\title{
A Web-based Information System for Plant Disease Forecast Based on Weather Data at High Spatial Resolution
}

\author{
Wee Soo Kang ${ }^{1}$, Soon Sung Hong ${ }^{2}$, Yong Kyu Han ${ }^{3}$ Kyu Rang Kim ${ }^{4}$, Sung Gi Kim ${ }^{2}$ and Eun Woo Park ${ }^{*}$ \\ ${ }^{1}$ Department of Agricultural Biotechnology, Seoul National University, Seoul 151-921, Korea \\ ${ }^{2}$ Gyeonggi-do Agricultural Research and Extension Services, Hwaseong 445-972, Korea \\ ${ }^{3}$ Epinet Corporation, Business Incubator, Seoul National University, Suwon 441-853, Korea \\ ${ }^{4}$ National Institute of Meteorological Research, Korea Meteorological Administration, Seoul 156-720, Korea \\ (Received on October 27, 2009; Accepted on November 18, 2009)
}

This paper describes a web-based information system for plant disease forecast that was developed for crop growers in Gyeonggi-do, Korea. The system generates hourly or daily warnings at the spatial resolution of $240 \mathrm{~m} \times 240 \mathrm{~m}$ based on weather data. The system consists of four components including weather data acquisition system, job process system, data storage system, and web service system. The spatial resolution of disease forecast is high enough to estimate daily or hourly infection risks of individual farms, so that farmers can use the forecast information practically in determining if and when fungicides are to be sprayed to control diseases. Currently, forecasting models for blast, sheath blight, and grain rot of rice, and scab and rust of pear are available for the system. As for the spatial interpolation of weather data, the interpolated temperature and relative humidity showed high accuracy as compared with the observed data at the same locations. However, the spatial interpolation of rainfall and leaf wetness events needs to be improved. For rice blast forecasting, $44.5 \%$ of infection warnings based on the observed weather data were correctly estimated when the disease forecast was made based on the interpolated weather data. The low accuracy in disease forecast based on the interpolated weather data was mainly due to the failure in estimating leaf wetness events.

Keywords : disease forecasting, infection risk map, weather interpolation

Disease forecasts can assist crop growers in determining when or if pesticides are sprayed. Efforts to provide the growers with plant disease forecasts based on weather data have been made in many studies (Campbell and Madden, 1990). In most cases, automated weather stations (AWS) are often used to collect weather data for plant disease forecast at the near real-time basis. However, use of AWS is

\footnotetext{
*Corresponding author.

Phone) +82-2-880-4672, FAX) +82-2-872-2317

E-mail)ewpark@snu.ac.kr
}

often limited due to the high costs for procurement and maintenance. Recent advances in information technology have provided various information delivery systems through which crop growers can have easy access to plant disease forecast information. For example, common use of personal computers with powerful computing resources, web-based Internet systems and wireless communication infrastructures allowed us to do instant data processes for collecting weather data from AWS's installed at remote places, analyzing huge data sets to generate disease forecast information and distributing the information to crop growers at near real-time basis (Kang et al., unpublished; Kim, 1995; Magarey et al., 1997; Magarey et al., 2007; Rajotte et al., 1992).

Application of geographic information systems (GIS) and simulation models for weather data analysis has also made significant contributions to improving temporal and spatial resolution of weather data, consequently making plant disease forecasts applicable to disease management in practice. Estimation of site-specific weather information is possible without on-site sensors using weather simulation models and GIS techniques (Magarey et al., 2001; Russo and Zack, 1997). The spatial resolution of mesoscale weather data can be enhanced in two ways: the localized mesoscale weather simulation (Seem et al., 2000) and the spatial interpolation (Seem et al., 1991). Although the localized weather simulation models are sophisticated and require a significant computing power, all weather elements interested could be estimated. In contrast, the spatial interpolation takes less time and computing power in calculation than the mesoscale weather simulation. The geostatistical analyses for spatial interpolation include kriging, inversedistance weighting (IDW), 2-D splines, trend-surface regression, and so on (Dodson and Marks, 1997; Myers, 1994). Correlation analysis was also used to estimate the maximum and minimum air temperatures at a spatial scale of individual farms using data from three weather stations (Seem et al., 1991). Correction for elevation often results in improvement in spatial interpolation of temperature, as was shown by Yun et al. (1999) who used IDW to estimate local 
air temperatures from synoptic observation data. For relative humidity, rainfall, and leaf wetness whose spatial variations are not as continuous as that of air temperature, data from remote sensing technologies, such as radar measurement and satellite imagery, are used to improve spatial interpolation (Anderson et al., 2001; Workneh et al., 2005).

At present, the Korea Meteorological Administration (KMA) operates two nationwide weather observation networks in Korea: the Synoptic Weather Observation Network (SWON) for standard meteorological observations, and the Automated Weather Station Network (AWSN) for monitoring weather disasters in Korea. The SWON and AWSN consist of 82 stations $(29.0 \pm 23.1 \mathrm{~km}$ apart $)$ and 627 stations (11.3 $\pm 6.8 \mathrm{~km}$ apart) nationwide, respectively, collecting at $60 \mathrm{sec}$ intervals, which are relatively dense in spatial and temporal scale in the world. In addition to SWON and AWSN, the Gyeonggi-do Agricultural Research and Extension Services (GARES) operates 26 AWS's installed in crop canopies in Gyeonggi-do, collecting at $60 \mathrm{sec}$ intervals with hourly and daily summaries.

In this study, we developed a web-based information system for plant disease forecast based on the real-time weather data from KMA. The system provides near realtime information on possible occurrence of plant diseases at the spatial resolution of $240 \mathrm{~m} \times 240 \mathrm{~m}$, so that crop growers can have disease forecast for their farm areas through Internet at their convenience.

\section{System Structure}

System hardware and software. The information delivery system operates on a server with dual CPU of $2.5 \mathrm{GHz}$ speed, 4 GB RAM, and 270 GB HDD running Linux operating system (Canonical Ltd.; http://www.ubuntu.com). MySQL (Sun Microsystems, Inc.; http://www.mysql.com) and Apache (The Apache Software Foundation; http:// www.apache.org) were used for database management server and web server, respectively. PHP (The PHP Group; http://www.php.net) and Java (Sun Microsystems, Inc.; http://java.sun.com) languages were used in programming applications. PHP was also used in web scripting.

System outline. The information delivery system has the internal structure, as shown in Fig. 1. The system consists of four sub-systems, such as weather data acquisition system (WDAS), job process system (JPS), data storage system (DSS), and web service system (WSS). The WDAS retrieves weather observation data from KMA and the AWS's installed by GARES, and stores the data to DSS. The JPS makes weather interpolation and disease forecast data by processing the weather observation data. The DSS stores both observed and interpolated weather data as well

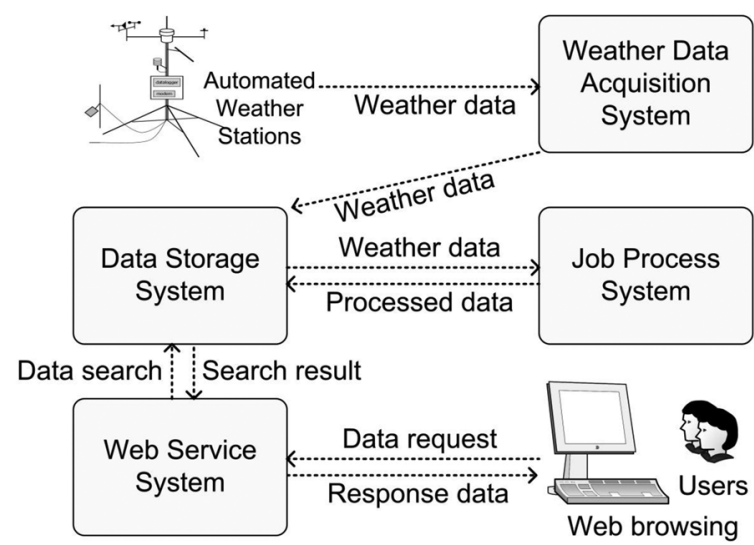

Fig. 1. Structure diagram of the overall disease forecasting system.

as disease forecast results, and provides the data to other sub-systems upon their requests. The WSS delivers the weather and disease forecast information to users via Internet web page.

Weather data acquisition system. Weather elements used by the forecasting system are hourly data on air temperature $\left({ }^{\circ} \mathrm{C}\right)$, relative humidity $(\%)$, and rainfall $(\mathrm{mm})$ from both SWON and AWSN of KMA, and the AWS's of GARES (Fig. 2). Among the AWS's operated by GARES, 16 stations were installed at rice paddy fields and 7 stations at pear orchards, 2 stations at apple orchards, and 1 station at grape vineyard to monitor weather conditions within canopies. The in-canopy AWS's (13.6 $\pm 4.1 \mathrm{~km}$ apart) collect hourly and daily weather data with a data logger (CR10X, Campbell Scientific, Logan, UT) measuring at 60 sec intervals. The station observes air temperature $\left({ }^{\circ} \mathrm{C}\right.$; HMP45C, Campbell Scientific), relative humidity (\%; HMP45C), rainfall (mm; TE525MM, Campbell Scientific), wind speed $(\mathrm{m} / \mathrm{s} ; 034 \mathrm{~A}$, Campbell Scientific), wind direction (degree, 034A), leaf wetness duration (hr/hr; 237 , Campbell Scientific), and solar radiation $\left(\mathrm{MJ} / \mathrm{m}^{2}\right.$; LI200X, Campbell Scientific).

Weather data files are transferred to the system from KMA in an ASCII text format. The transferred data include regional weather forecasts by KMA as well as SWON and AWSN observations. The KMA Data File Processor in WDAS is run every 10 minute to read the files and saves the weather data in DSS. The weather data observed by the in-canopy AWS's of GARES are retrieved in two ways: 1) the Weather Station Dialer dials to the stations having wired telephone modem every hour and collects weather data logged in the data logger; 2) the stations having wireless CDMA modem make connection to Weather Data Server Daemon (WDSD) in WDAS via wireless Internet connection every hour. Then, WDSD retrieves weather data from the stations and stores them in DSS. 


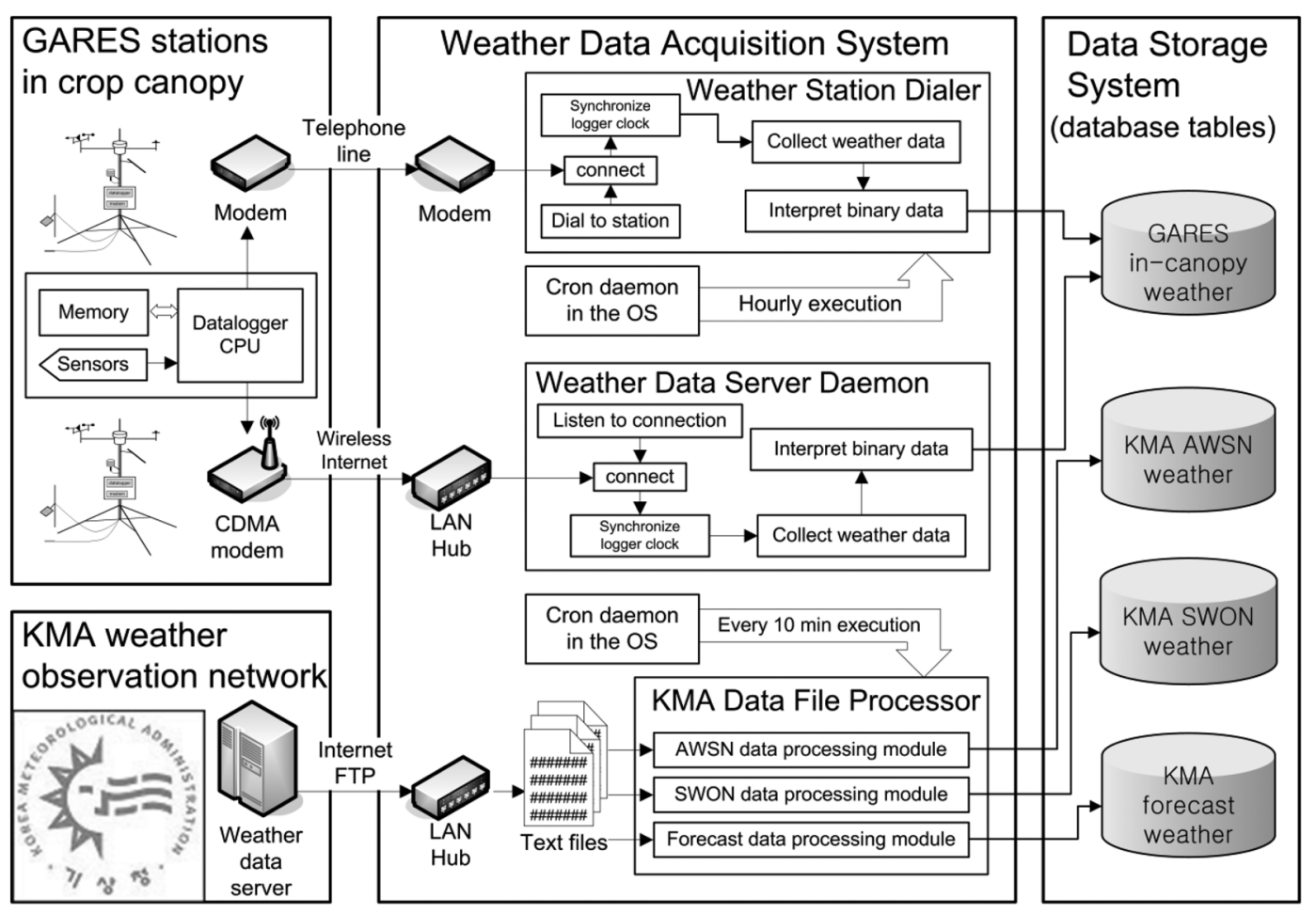

Fig. 2. Structure diagram of the weather data acquisition system (WDAS).

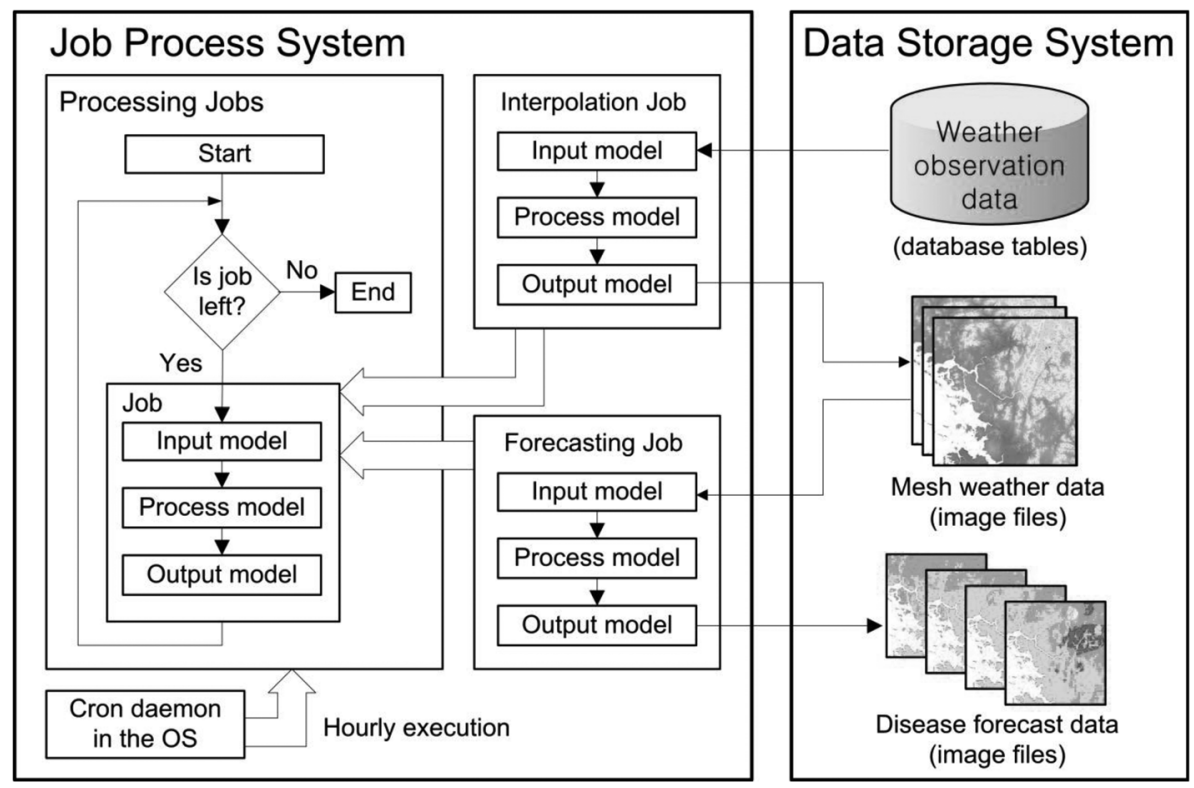

Fig. 3. Structure diagram of the job process system (JPS) for interpolation of weather data, disease forecast, and map image drawing.

Job process system. The JPS carries out data processing and calculations to produce output data that will be shown to users (Fig. 3). The interpolation job produces hourly mesh weather data at the spatial resolution of $240 \mathrm{~m} \times 240 \mathrm{~m}$ using observed hourly weather data from AWSN. If the AWSN data are not available at the moment of interpolation due to missing or delay, the SWON data or the incanopy weather observation data from GARES are used to ensure real-time forecasting. The system covers only Gyeonggi-do, so it uses the weather data monitored by the stations in Gyeonggi-do and its surrounding areas (191 AWSN stations, 21 SWON stations, and 26 in-canopy stations, as shown in Fig. 4).

Estimation of air temperature, relative humidity, and rainfall for mesh grids is made by the inverse squared distance weighting (IDW) method with observations from 


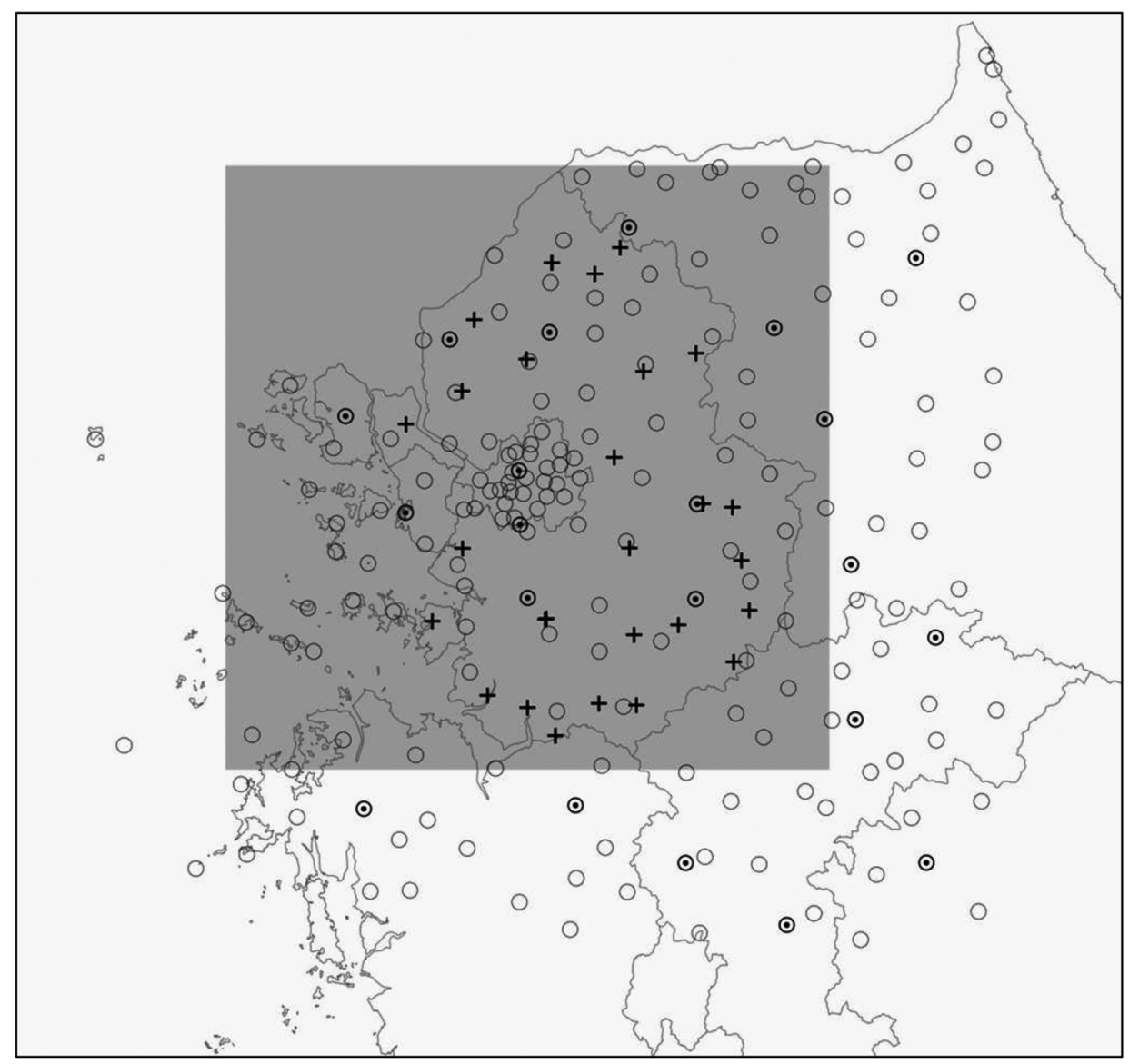

Fig. 4. Locations of automated weather stations in Gyeonggi-do. Circles $(\odot)$ and dotted circles $(\odot)$ indicate the stations in Synoptic Weather Observation Network and Automated Weather Stations Network, respectively, operated by the Korea Meteorological Administration. Crosses $(+)$ indicate the stations in rice paddy fields of the Gyeonggi-do Agricultural Research and Extension Services. Shaded region is the target area of weather interpolation and disease forecasting in the system.

neighboring stations (Yun et al., 1999). Although the number of neighbors more than 6 have little effect on the interpolation (Dodson and Marks, 1997), we included all the stations available in the interpolation to reduce coarse results. Thus, point weather observations of about $10 \mathrm{~km}$ $(10.1 \pm 5.3 \mathrm{~km}$ for AWSN stations in Gyeonggi-do and its surrounding areas) apart from each other are downscaled into the mesh weather of $240 \mathrm{~m} \times 240 \mathrm{~m}$ resolution. As for air temperature, correction was applied after downscaling as the following equation (Yun et al., 1999):

$$
\mathrm{T}=\frac{\sum_{i} \frac{T_{i}}{d_{i}^{2}}}{\sum_{i} \frac{1}{d_{i}^{2}}}-\gamma\left(z-\frac{\sum_{i} \frac{z_{i}}{d_{i}^{2}}}{\sum_{i} \frac{1}{d_{i}^{2}}}\right)
$$

where $T_{i}=$ air temperature at the $i^{\text {th }}$ neighbor, $d_{i}=$ distance between the site and the $i^{\text {th }}$ neighbor, $z_{i}=$ elevation of the $i^{\text {th }}$ neighbor, $\mathrm{z}=$ elevation of the site, and $\gamma=$ the lapse rate of air temperature decrement as elevation increases. The global mean of $\gamma\left(-0.0065^{\circ} \mathrm{C} \mathrm{m}^{-1}\right)$ was used in this study (Barry and Chorley, 2003).

In the cases of relative humidity and rainfall, the IDW method was also applied to downscale the data without the correction for elevation. Since leaf wetness duration is not available in the data from SWON and AWSN, it was estimated by the simple relative humidity model (Wilks and Shen, 1991; Yun et al., 1998) as follows using the interpolated rainfall and relative humidity data;

$$
\begin{aligned}
& \text { leaf wetness duration }= \\
& \begin{cases}1, & \text { if rainfall } \geq 0.1 \mathrm{~mm} \text { or relative humidity }>95 \% \\
0, & \text { if otherwise }\end{cases}
\end{aligned}
$$

The forecasting job calculates infection risks using the mesh weather data. The results have the same spatial resolution as the mesh weather data and are stored in DSS. All the jobs in JPS are processed every hour.

Data storage system. The DSS stores data as database 


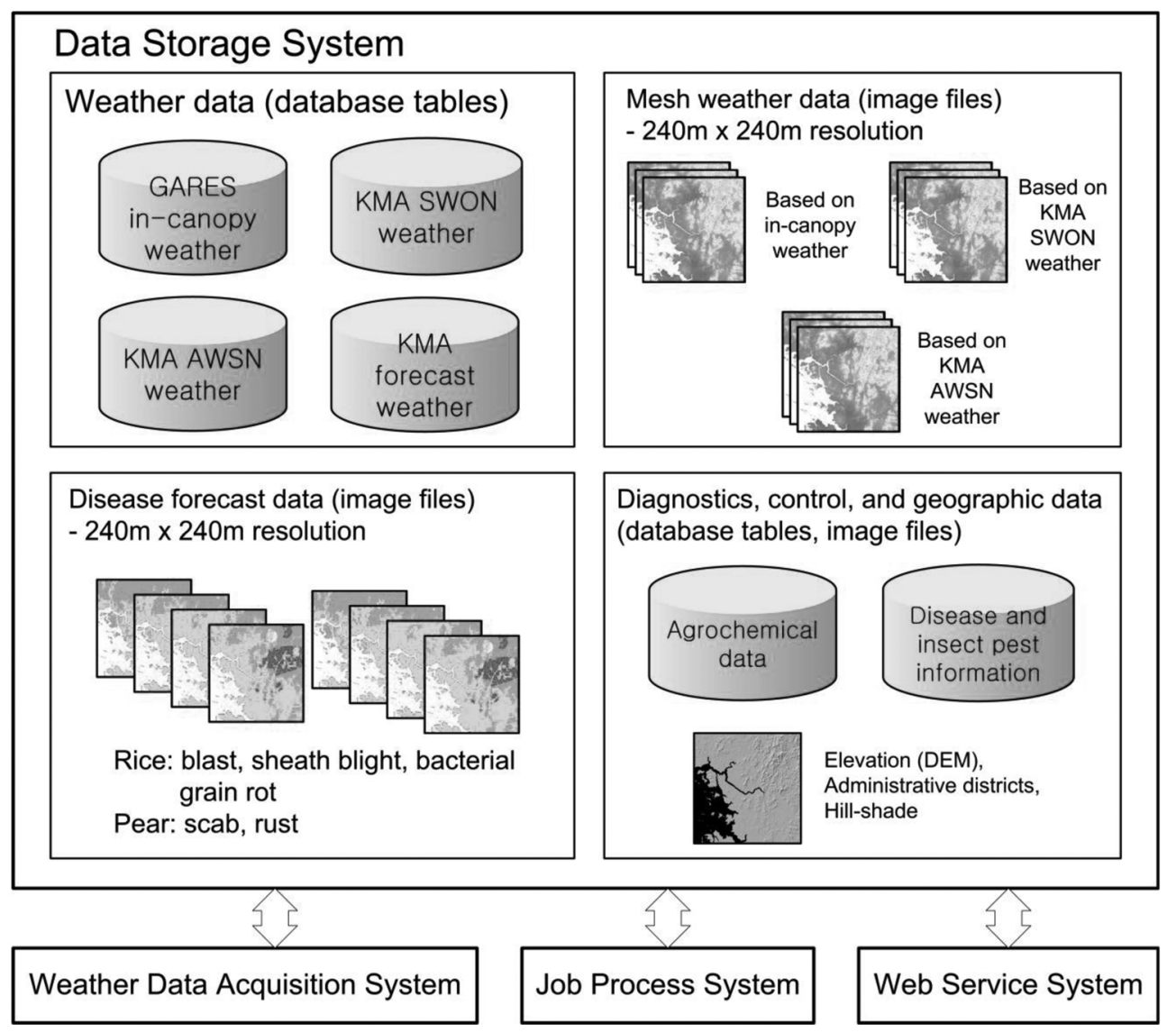

Fig. 5. Structure diagram of the data storage system (DSS).

tables or files (Fig. 5). Weather data from SWON, AWSN, and the in-canopy AWS's of GARES are stored in DSS as database tables. Interpolated weather data and disease forecast data resulted from JPS are stored as image files so as to be shown in the web browser immediately upon requests. The DSS provides all the data to the other subsystems on demand. The DSS also stores the static raster data that are maps of administrative boundary, elevation, and hill-shade. In addition, DSS stores database tables of application guide for agricultural chemicals and detailed information on diagnosis and control of crop diseases and insect pests.

Web service system. The WSS interacts with users through web browsers to provide information upon users' requests (Fig. 6). The WSS presents the interpolated weather and disease forecast data through the web map interface. In addition, WSS provides information on diseases, pests, control, and chemicals in detail.

Disease forecasting model. The Yoshino model (Yoshino, 1979) was used to forecast rice leaf blast infection. It determines infection periods of the pathogen (Magnaporthe grisea) based on weather conditions. The model evaluates weather conditions every hour and produces hourly results regarding whether or not the conditions for successful infections could have occurred. The conditions for successful infection determined by the model are as follows;

1) the moving average of air temperature during past 5 days is $20 \sim 25^{\circ} \mathrm{C}$

2) rainfall is below $4 \mathrm{~mm} / \mathrm{hr}$, and

3 ) the continuous wet hours exceeds 4 hours more than the base wet hours calculated by the equation:

Base wet hours $=60.09-4.216 \times$ temp $_{\text {wet }}+0.08858 \times$ temp $_{\text {wet }}^{2}$,

where temp $p_{\text {wet }}$ is air temperature while leaves are wet.

The infection hours determined by the model were accumulated for a day to calculate the daily infection warning hours (DIWH; hr). DIWH was categorized into four risk levels: $\rightarrow$ Zero (DIWH=0 hr) “ $\rightarrow$ ', “ $\rightarrow$ ' Low (1 $\mathrm{hr} \leq \mathrm{DIWH}<3 \mathrm{hr})$ " $\rightarrow$ ', “ $\rightarrow$ ' Intermediate $(3 \mathrm{hr} \leq \mathrm{DIWH}$ $<6 \mathrm{hr}$ ) " $\rightarrow$ ', and ' $\rightarrow$ ' High $(\mathrm{DIWH} \leq 6 \mathrm{hr})$ ' $\rightarrow$ '.

Verification of interpolated weather data. The interpolation of weather data at the spatial resolution of 240 


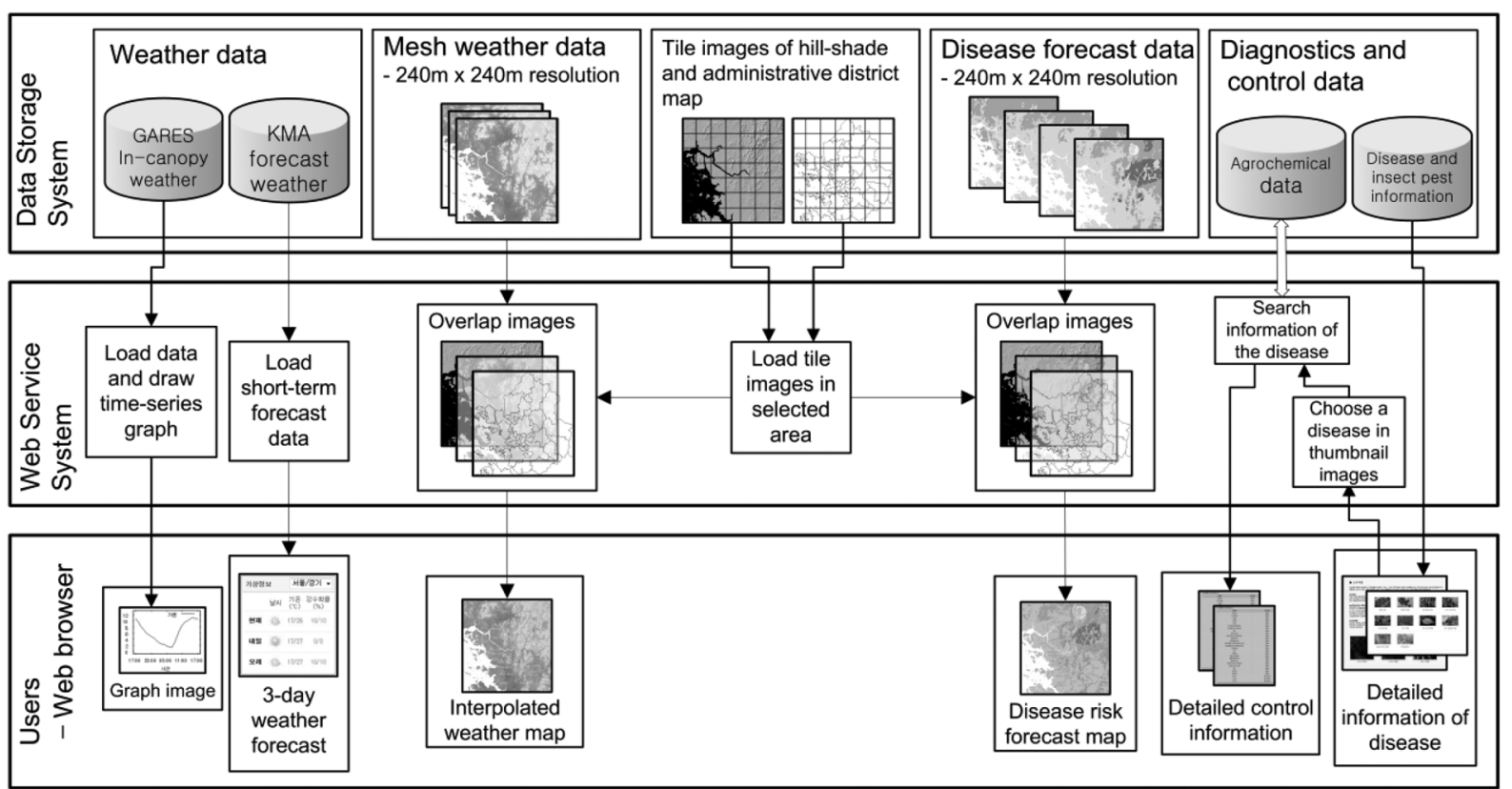

Fig. 6. Structure diagram of the web service system (WSS).

$\mathrm{m} \times 240 \mathrm{~m}$ was evaluated for its accuracy by comparing AWS data with the interpolated data for the locations where AWS's were installed. The observed weather data were obtained from 10 AWS's installed at rice paddy fields by GARES (Table 1). A total of 110,189 sets of observed and interpolated hourly data for 10 locations during the period of May $1^{\text {st }}$ to October $20^{\text {th }}$ in 2005-2007 were used for comparison. For data verification, the linear regression analysis between the interpolated and the observed data was used for air temperature and relative humidity. In the cases of rainfall and leaf wetness events, the two-way contingency table analysis was used (Sokal and Rohlf, 1973). In the two-way contingency table analysis, two categorical measures of accuracy for event forecast were calculated. The probability of detection (POD) is the percent of events that are forecast, and the false alarm ratio (FAR) is a measure of the failure of the forecaster to exclude non-event cases (Donaldson et al., unpublished; Schaefer, 1990). A rainfall event was considered to occur in an hour when rainfall $\geq 0.1 \mathrm{~mm} / \mathrm{hr}$, and leaves were considered to be wet in an hour when the leaf wetness period was at least one minute during that hour.

Verification of rice blast forecasts. The accuracy in infection risk forecast based on the interpolated weather data was evaluated for the period of July $15^{\text {th }} \sim$ August $15^{\text {th }}$, in 2005-2007 during which weather conditions were often favorable for leaf blast development in Korea. The DIWH for rice blast calculated based on the interpolated weather data were compared with those based on the observed weather data. The DIWH for the total of 94 days
Table 1. Geographic locations of rice paddy fields in Gyeonggido, Korea where automated weather stations were installed to monitor hourly weather conditions within the rice canopy. The monitored data were used to verify the estimated weather data by spatial interpolation based on the AWSN data

\begin{tabular}{cccc}
\hline \hline Location & Longitude $\left({ }^{\circ}\right)$ & Latitude $\left({ }^{\circ}\right)$ & Elevation $(\mathrm{m})$ \\
\hline Anseong & 127.3057556 & 37.0115639 & 34 \\
Gapyeong & 127.5015889 & 37.8438861 & 68 \\
Gimpo & 126.6289944 & 37.6841778 & 25 \\
Icheon & 127.4341667 & 37.1997889 & 63 \\
Paju & 126.7979611 & 37.7619500 & 36 \\
Paltan & 126.9062306 & 37.1532917 & 27 \\
Pocheon & 127.2789278 & 38.0974028 & 125 \\
Siheung & 126.7947500 & 37.3887083 & 32 \\
Taean & 127.0392308 & 37.2212375 & 45 \\
Yeoncheon & 127.0723750 & 38.0639500 & 60 \\
\hline
\end{tabular}

in 2005-2007 from 10 locations were categorized into four risk levels as described previously. The correspondence of daily risk levels based on the interpolated and observed weather data was evaluated and the categorical measures such as POD and FAR were calculated for the events of risk levels which belonged to Zero, $\geq$ Low, $\geq$ Intermediate, and High.

\section{Results}

Disease forecasting system. The web-based disease forecasting system can be found at http://www.epilove.com (Fig. 7). The information available in the system includes hourly and daily weather data, and daily forecasts on 

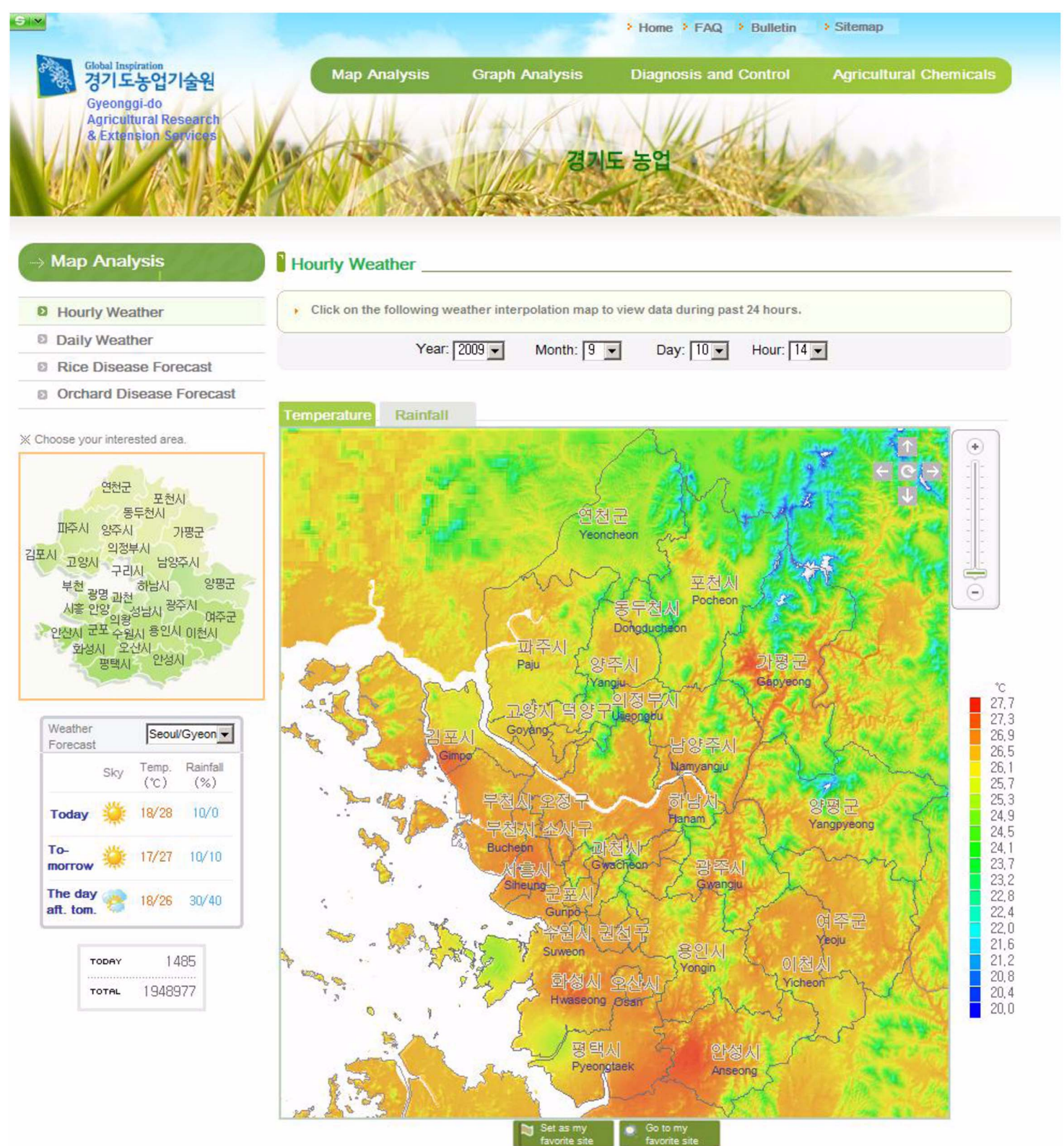

경기도도농업기술월 $\mid 315$ Gisan-dong, Hwaseong-si, Gyeonggi-do, Republic of Korea Tel: +82-31-229-6114

Copyright (C) 2009 by Gyeonggi-do Agricultural Research \& Extension Services. All rights reserved

Fig. 7. The home page screen shot of the disease forecasting system: http://www.epilove. Contents of the website are in Korean to be understandable to crop growers in Korea. In this figure, the contents were partially translated into English for the convenience of readers.

diseases of rice (blast, sheath blight, and bacterial grain rot) and pear (rust and scab) in map images and graphic forms. The map images of weather and disease forecast data are at the spatial resolution of $240 \mathrm{~m} \times 240 \mathrm{~m}$. In addition, 3-day forecast on synoptic weather conditions is available for regional areas of Korea. The map images can be panned across the geographic area of Gyeonggi-do, and zoomed in and out, showing the data layer overlapped by the layers of hill-shade and administrative boundary for users to find the points of interest easily. For each grid point on the map image, users can have details of weather and disease forecast information during the past 15 days (Fig. 8). The system also provides diagnosis and control information on 450 diseases and 183 insect pests of 117 crops, and usages of registered agricultural chemicals.

Verification of interpolated weather data. Accuracy of the weather interpolation at the spatial resolution of 240 $m \times 240 m$ was evaluated by comparing 110,189 sets of the observed weather data from GARES and the hourly data 


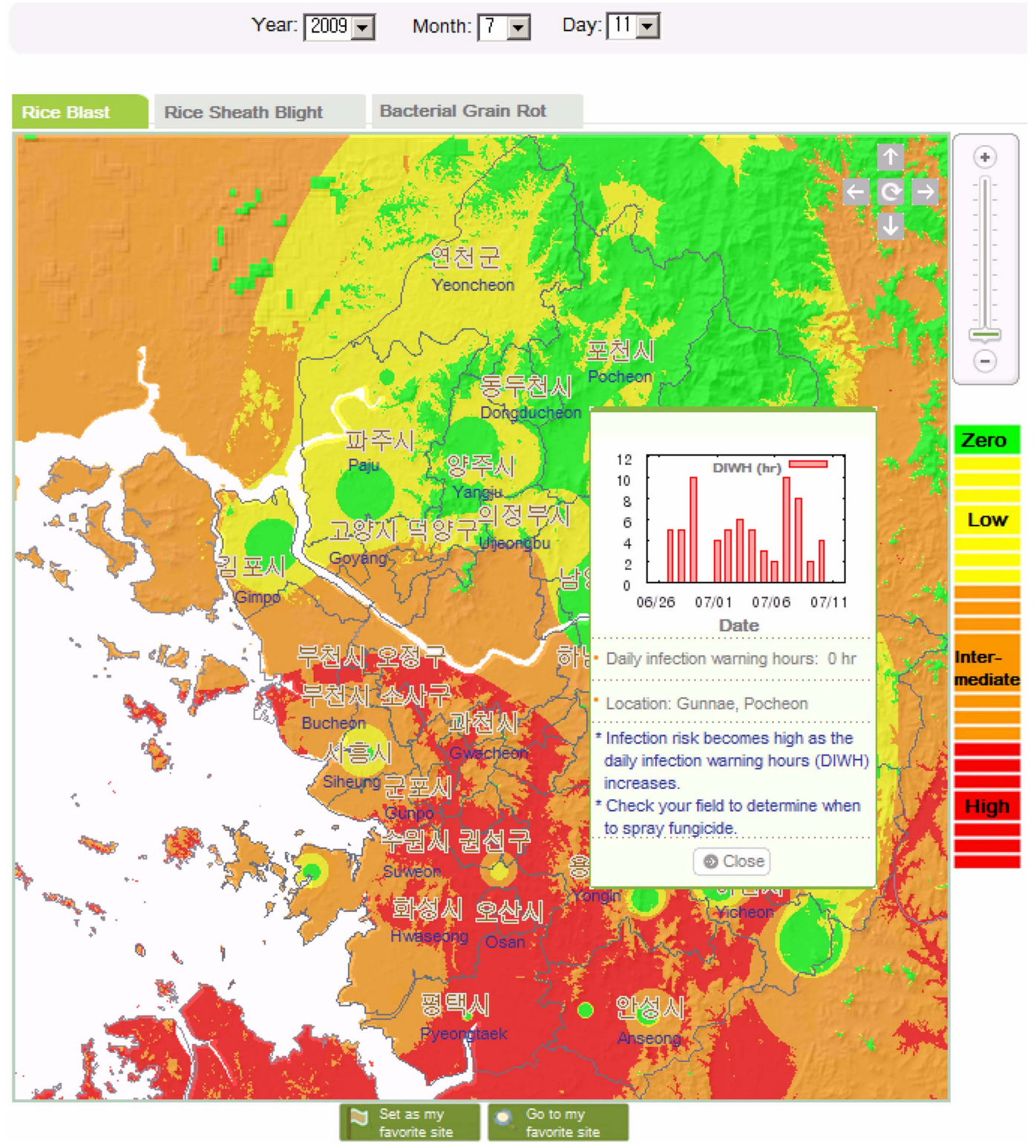

Fig. 8. An example of rice blast forecast by the disease forecasting system. The spatial resolution of disease forecast is $240 \mathrm{~m} \times 240 \mathrm{~m}$, and information on daily infection risks of past 15 days is available for each grid on the map. Contents of the website area in Korean to be understandable to crtop growers in Korea. In this figure, the contents were partially translated into English for the convenience of readers.

interpolated from the AWSN data. The interpolated air temperatures appeared similar to the observed data. The regression analysis between the interpolated and observed temperatures resulted in $\mathrm{y}=2.73+0.98 \mathrm{x}\left(\mathrm{R}^{2}=0.96\right)$, where $\mathrm{y}$ and $\mathrm{x}$ are interpolated and observed data, respectively (Fig. 9A). The regression analysis indicated slight overestimation for low temperatures, which often occurred at night and early morning (Fig. 9B).

Downscaling of relative humidity by spatial interpolation resulted in relatively poor estimation as compared with the case of air temperature (Fig. 9C). The relationship between the interpolated $(\mathrm{y})$ and the observed $(\mathrm{x})$ relative humidity was described by $y=-0.22+0.94 \times$. Although the coefficient of determination $\left(\mathrm{R}^{2}\right)$ suggested that $86 \%$ of variations in interpolated relative humidity was accounted for by the observed relative humidity, the deviation from expected relative humidity became greater as the observed relative humidity increased, and a large number of outliers were noticed in the data. Underestimation of relative humidity was found in the majority of interpolated data throughout 24 hours a day (Fig. 9D).

The results of two-way contingency table analysis for rainfall and leaf wetness events are shown in Table 2. Rainfall events have occurred in $11.2 \%$ cases of 110,189 

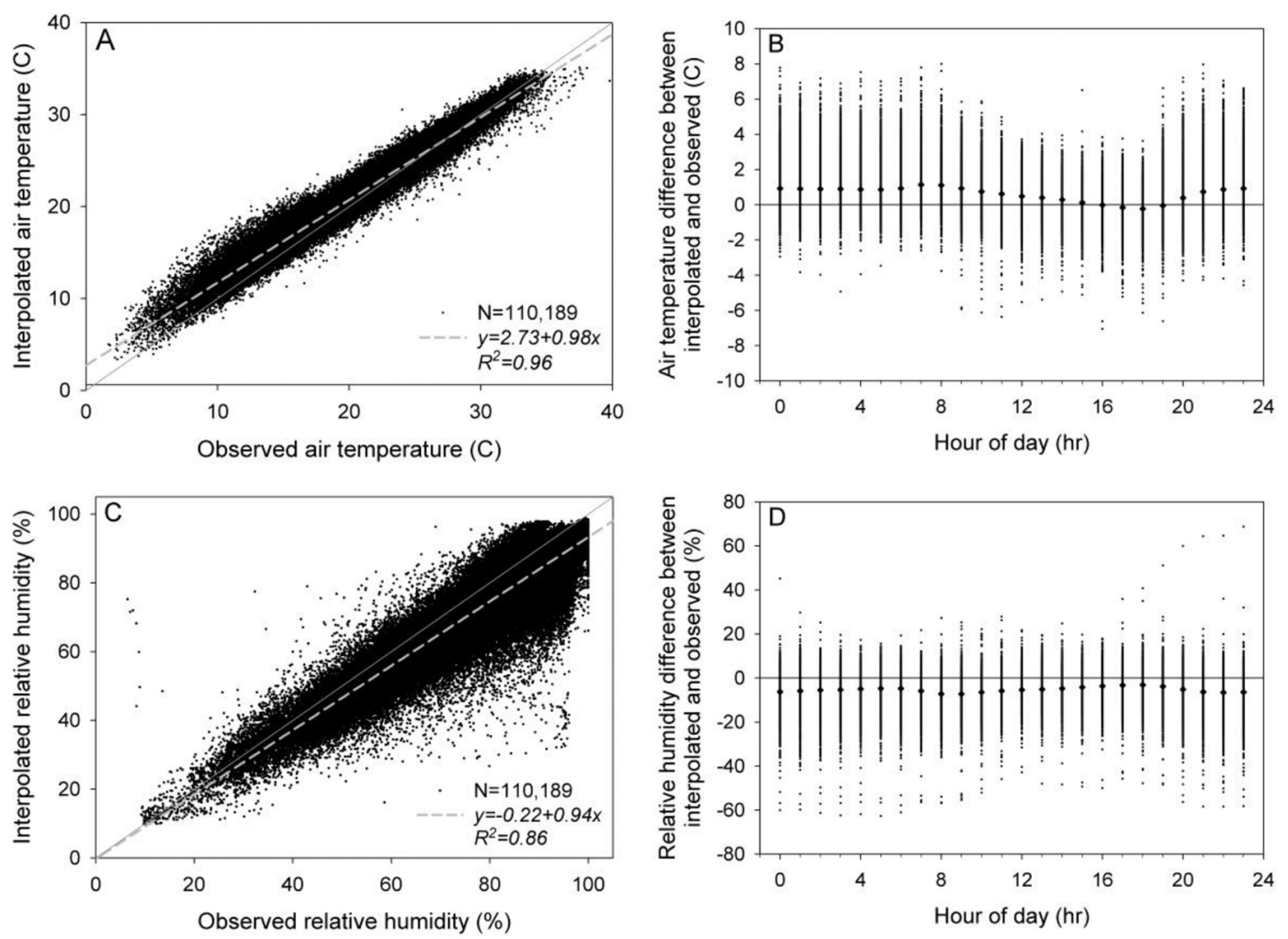

Fig. 9. The relationship between the observed and interpolated data on hourly air temperature and relative humidity during the period of May $1^{\text {st }} \sim$ October $20^{\text {th }}$ in 2005-2007. The observed data were collected by the automated weather stations in rice paddy fields of the Gyeonggi-do Agricultural Research and Extension Services. The interpolated data were from the AWSN operated by Korea Meteorological Administration. A and C, a scatter diagram of observed and interpolated data; B and D, hourly differences between the observed and the interpolated data (=interpolated-observed) during 24 hours a day. The symbol $\bullet$ indicates the average of differences for each hour.

Table 2. Results of the two-way contingency table analysis to evaluate accuracy of spatial interpolation on rainfall and leaf wetness events as compared with the observed data from 10 locations $s^{\mathrm{a}}$ during the period of May $1^{\text {st }} \sim$ October $20^{\text {th }}$ in 2005-2007. A total of 110,189 data points were included in the comparison

\begin{tabular}{lcc}
\hline \hline \multirow{2}{*}{ Index $^{\mathrm{b}}$} & \multicolumn{2}{c}{ Categorical score $^{\mathrm{c}}(\%)$} \\
\cline { 2 - 3 } & Rainfall $^{\mathrm{d}}$ & Leaf wetness $^{\mathrm{d}}$ \\
\hline Hit & 9.0 & 21.9 \\
Miss & 2.3 & 31.6 \\
\hline False alarm & 5.7 & 2.3 \\
Correct rejection & 83.1 & 44.2 \\
\hline Probability of detection (POD) & 79.8 & 41.0 \\
False alarm ratio (FAR) & 38.7 & 9.5 \\
\hline
\end{tabular}

${ }^{\text {a The }} 10$ locations are described in Table 1.

${ }^{\mathrm{b}} \mathrm{Hit}$, miss, false alarm, and correct rejection are relative frequency that event occurred in both the observed and the interpolated, event occurred in the observed but it did not occur in the interpolated, event did not occur in the observed but it occurred in the interpolated, and event did not occur in both the observed and the interpolated, respectively. $\mathrm{POD}=\mathrm{Hit} /(\mathrm{Hit}+\mathrm{Miss}) . \mathrm{FAR}=\mathrm{False}$ alarm/(Hit + False alarm)

${ }^{\mathrm{c}}$ All the scores were rounded off to one decimal place.

${ }^{\mathrm{d}}$ Rainfall and leaf wetness events mean rainfall $\geq 0.1 \mathrm{~mm} / \mathrm{hr}$ and leaf wetness duration $>0 \mathrm{hr} / \mathrm{hr}$, respectively. data points. However, the interpolation hit in the cases of $9.0 \%$, and missed $2.3 \%$ cases. Consequently, the probability of detection (POD) by the spatial interpolation was $79.8 \%$ of rainfall events (rainfall $\geq 0.1 \mathrm{~mm}$ ). The spatial interpolation resulted in false alarm for rainfall events in $5.7 \%$ cases, and the false alarm ratio (FAR) was $38.7 \%$. The FAR is the percent of rainfall event estimation that fail to materialize.

As for leaf wetness, $53.5 \%$ of 110,189 data points indicated that leaf wetness has occurred, but the spatial interpolation has missed leaf wetness events in $31.6 \%$ cases (Table 2). The POD and FAR for leaf wetness estimation were $41.0 \%$ and $9.5 \%$, respectively, suggesting that $41.0 \%$ of leaf wetness events was correctly estimated and $9.5 \%$ of no-wetness (dry) events was incorrectly estimated by the spatial interpolation.

Verification of rice blast forecasts. The result of two-way contingency table analysis for rice blast forecasts are shown in Table 3. During 94 days in 2005-2007, 79.7\% cases of 940 data points were determined to be unfavorable for infection (DIWH=Zero) and $20.3 \%$ cases were determined 
Table 3. Results of two-way contingency table analysis to evaluate accuracy of rice blast forecasts based on the interpolated weather data as compared with the forecasts based on the observed data from 10 locations $^{\mathrm{a}}$ during the period of July $15^{\text {th }} \sim$ August $15^{\text {th }}$ in 2005-2007. A total of 940 data points were included in the comparison

\begin{tabular}{lrrcr}
\hline \multirow{2}{*}{ Index $^{\mathrm{b}}$} & \multicolumn{4}{c}{ Categorical score $^{\mathrm{c}}(\%)$} \\
\cline { 2 - 5 } & Zero $^{\mathrm{d}} \geq$ Low $^{\mathrm{d}}$ & $\begin{array}{c}\geq \text { Inter- }^{\text {mediate }} \\
\text { med }^{\mathrm{d}}\end{array}$ & High $^{\mathrm{d}}$ \\
\hline Hit & 78.0 & 9.0 & 5.0 & 2.0 \\
Miss & 1.7 & 11.3 & 7.9 & 3.9 \\
\hline False alarm & 11.3 & 1.7 & 1.3 & 1.3 \\
Correct rejection & 9.0 & 78.0 & 85.9 & 92.8 \\
\hline Probability of detection (POD) & 97.9 & 44.5 & 38.8 & 33.9 \\
False alarm ratio (FAR) & 12.6 & 15.8 & 20.3 & 38.7 \\
\hline
\end{tabular}

${ }^{\text {a }}$ The 10 locations are described in Table 1.

${ }^{\mathrm{b}} \mathrm{Hit}$, miss, false alarm, and correct rejection are relative frequency that event occurred in both the observed and the interpolated, event occurred in the observed but it did not occur in the interpolated, event did not occur in the observed but it occurred in the interpolated, and event did not occur in both the observed and the interpolated, respectively. $\mathrm{POD}=\mathrm{Hit} /(\mathrm{Hit}+\mathrm{Miss}) . \mathrm{FAR}=$ False alarm/(Hit+False alarm).

${ }^{c}$ All the scores were rounded off to one decimal place.

${ }^{\mathrm{d}}$ The events Zero, $\geq$ Low, $\geq$ Intermediate, and High mean that the levels of rice blast forecasts are Zero, greater than or equal to Low, greater than or equal to Intermediate, and High, respectively.

to be favorable for infection (DIWH $\geq$ Low) by the Yoshino model based on observed weather data. For higher infection risk levels, warnings of DIWH $\geq$ Intermediate and DIWH= High occurred only in the cases of $12.9 \%$ and $6.0 \%$, respectively. Warnings based on the interpolated weather data hit the warnings based on the observed weather data up to $9.0 \%, 5.0 \%$, and $2.0 \%$, and missed $11.3 \%, 7.9 \%$, and $3.9 \%$, respectively, for the risk levels of DIWH $\geq$ Low, $\mathrm{DIWH} \geq$ Intermediate, and DIWH=High. The probability of detection (POD) of the forecast based on the interpolated weather data was up to $97.9 \%, 44.5 \%, 38.8 \%$, and $33.9 \%$, for the risk levels of DIWH=Zero, DIWH $\geq$ Low, DIWH $\geq$ Intermediate, and DIWH=High, respectively. The FAR was also more frequent for higher infection risk levels than for low or no infection risk levels (Table 3 ).

\section{Discussion}

We aimed to develop and implement an information system for disease and insect pest forecast with a special consideration on practical use by crop growers. In addition, information on diagnosis, ecological characteristics, and control chemicals of diseases and insect pests is available in the system to add to the practical value. The major characteristics that make the information system practically useful to crop growers include 1) accuracy, 2) site-specificity, and 3) near real-time delivery of disease forecasts, and 4) easy accessibility to the system. The accuracy is mainly dependent upon validity of disease forecasting model and accuracy of weather data. The Yoshino model (Yoshino, 1979) has been tested intensively in Korea and showed acceptable performance in estimating infection periods of rice blast during 1993-1996 (Kim, 2000; Park et al., unpublished). Accuracy of weather data can be assured by accurate measurement of weather conditions. In this study, the original weather data were from AWSN of KMA which has more than 600 AWS's covering about 100,000 $\mathrm{km}^{2}$ (Joo et al., 2005). The high spatial density of AWS's and the data quality control system of KMA ensure the quality of weather data in Korea. However, the AWS's are often installed at non-agricultural areas or on the roof-top of buildings (Joo et al., 2005), so that the observed weather data from such AWS's could not represent the microclimate conditions within crop plant canopy. The discrepancies between the observed and the interpolated air temperature and relative humidity in this study may be partly due to the inadequate sites of weather monitoring in AWSN or errors in observed data from AWS's in the paddy fields.

The spatial resolution of $240 \mathrm{~m} \times 240 \mathrm{~m}$ was high enough to generate site-specific disease forecasts for small scale farms in Korea. However, except for elevation for the air temperature interpolation, the IDW method of spatial interpolation did not consider factors affecting local atmospheric phenomena such as land topography, land cover, slope, aspect, surface water, etc (Dodson and Marks, 1997; Yun, 2000). Most areas in Korea have complicated topography or mixed land cover, so that we cannot ensure the accuracy of weather estimation at high spatial resolution using only the spatial interpolation. The interpolation method needs to be improved by incorporating the factors affecting local atmospheric fluctuations (Geiger, 1965; Magarey et al., 2001). In this study, the limited success in estimating rainfall and leaf wetness events may have resulted from the spatial discontinuity of rainfall events and the lack of physical consideration in the spatial interpolation. Adopting mesoscale weather simulation models may be an alternative strategy to enhance the accuracy of weather estimates at high spatial resolution (Kaplan et al., 1982; Kim et al., 2005; Manobianco et al., 1996; Seem et al., 2000), and consequently the accurate disease forecasts. For better estimation of leaf wetness events, the classification and regression tree (CART) models and the fuzzy logic models may be worth trying for improvement in plant disease forecasting (Kim et al., 2006).

We achieved the real-time generation and delivery of disease forecasting information by automating all the procedures from the weather data collection to the web service. No user inputs are required for the system operation. The high density of AWS's and the wired and wireless network 
for data communication in Korea were the important technological infrastructure for the web-based real-time information system, and provided an excellent opportunity to develop a plant disease forecasting system that can be used in practice by crop growers.

Ease of access is an important issue for the utility of information system. Even though the forecast is correct, the forecast information could be ignored if users have difficulties in accessing and understanding the information due to delayed delivery or incomprehensibility. Subsequently the system loses an opportunity of validation and further improvement (Magarey et al., 2002). To make the information system user-friendly for local users in Korea, the weather and disease forecast data are provided in graphs, map images, and text forms in Korean. Especially, all map images are created as soon as the weather and disease forecast data are available and stored in the data storage system in order to instantly provide the map images upon requests by users. The system provides a disease risk map with administrative boundary map and hill-shade map to help users find their sites of interest. We hope that overlapping the risk map with satellite images, aerial photographs, and cadastral maps will enhance the usage of the system in the future.

\section{Acknowledgements}

This work was supported by a grant (0525-2006005) from Gyeonggi-do Agricultural Research and Extension Services, Gyeonggi, Republic of Korea in 2006.

\section{References}

Anderson, M. C., Bland, W. L. and Norman, J. M. 2001. Canopy wetness and humidity prediction using satellite and synopticscale meteorological observations. Plant Dis. 85:1018-1026.

Barry, R. G. and Chorley, R. J. 2003. Atmosphere, weather and climate. Routledge, London. $472 \mathrm{pp}$.

Campbell, C. L. and Madden, L. V. 1990. Forecasting plant diseases. In: Introduction to plant disease epidemiology: John Wiley \& Sons, Inc., New York, USA.

Dodson, R. and Marks, D. 1997. Daily air temperature interpolated at high spatial resolution over a large mountainous region. Clim. Res. 8:1-20.

Geiger, R. 1965. The climate near the ground. Harvard University Press, Cambridge, MA.

Joo, H. D., Lee, M. J. and Ham, I. W. 2005. The characteristics of air temperature according to the location of automatic weather system. J. Atmosphere 15:179-186.

Kaplan, M. L., Zack, J. W., Wong, V. C. and Tucillo, J. J. 1982. Initial results from a mesoscale atmospheric simulation system and comparisons with an AVE-SESAME I data set. Monthly Weather Review 110:1564-1590.
Kim, K. R. 1995. Development of a rice blast forecasting system based on near real-time microclimatic data, M. S. Thesis, Seoul National University, Suwon, Korea.

Kim, K. R. 2000. Weather-driven models for rice leaf blast and their implementation to forecast disease development on the near real-time basis. PhD Thesis, Seoul National University, Suwon, Korea.

Kim, K. R., Seem, R. C., Park, E. W., Zack, J. W. and Magarey, R. D. 2005. Simulation of grape downy mildew development across geographic areas based on mesoscale weather data using supercomputer. Plant Pathol. J. 21:111-118.

Kim, K. S., Gleason, M. L. and Taylor, S. E. 2006. Forecasting site-specific leaf wetness duration for input to disease-warning systems. Plant Dis. 90:650-656.

Magarey, P. A., Emmett, R. W., Herrmann, N. I., Wachtel, M. F. and Travis, J. W. 1997. Development of AusVit, a computerized decision support system for integrated management of diseases, pests and other production factors in Australian viticulture. Vitic. Enol. Sci. 52:175-179.

Magarey, R. D., Fowler, G. A., Borchert, D. M., Sutton, T. B., Colunga-Garcia, M. and Simpson, J. A. 2007. NAPPFAST: An Internet system for the weather-based mapping of plant pathogens. Plant Dis. 91:336-345.

Magarey, R. D., Seem, R. C., Russo, J. M., Zack, J. W., Waight, K. T., Travis, J. W. and Oudemans, P. V. 2001. Site-specific weather information without on-site sensors. Plant Dis. 85:1216-1226.

Magarey, R. D., Travis, J. W., Russo, J. M., Seem, R. C. and Magarey, P. A. 2002. Decision support systems: Quenching the thirst. Plant Dis. 86:4-14.

Manobianco, J., Zack, J. W. and Taylor, G. E. 1996. Workstationbased real-time mesoscale modeling designed for weather support to operations at the Kennedy Space Center and Cape Canaveral Air Station. Bull. Am. Meteor. Soc. 77:653-672.

Myers, D. E. 1994. Spatial interpolation: an overview. Geoderma 62:17-28.

Rajotte, E. G., Bowser, T., Travis, J. W., Crassweller, R. M., Musser, W., Laughland, D. and Sachs, C. 1992. Implementation and adoption of an agricultural expert system: The Penn State Apple Orchard Consultant (PSAOC). Acta Hortic. 313:227-231.

Russo, J. M. and Zack, J. W. 1997. Downscaling GCM output with a mesoscale model. J. Envir. Management 49:19-29.

Schaefer, J. T. 1990. The critical success index as an indicator of warning skill. Weather Forecast 5:570-575.

Seem, R. C., Magarey, R. D., Zack, J. W. and Russo, J. M. 2000. Estimating disease risk at the whole plant level with General Circulation Models. Environ. Pollut. 108:389-395.

Seem, R. C., Magnus, H. A. and Hjonnevaag. 1991. High resolution weather information for plant protection. EPPO Bull. 21:355-364.

Sokal, R. R. and Rohlf, F. J. 1973. Introduction to biostatistics. W. H. Freeman and Company, San Francisco. 368 pp.

Wilks, D. S. and Shen, K. W. 1991. Threshold relative humidity duration forecasts for plant disease prediction. J. Appl. Meteorol. 30:463-470. 
Workneh, F., Narasimhan, B., Srinivasan, R. and Rush, C. M. 2005. Potential of radar-estimated rainfall for plant disease risk forecast. Phytopathology 95:25-27.

Yoshino, R. 1979. Ecological studies on penetration of rice blast fungus, Pyricularia oryzae, into leaf epidermal cells. Bull. Hokuriku Agric. Exp. Stn. 22:163-221.

Yun, J. I. 2000. Estimation of climatological precipitation of North Korea by using a spatial interpolation scheme. Korean J. Agric. Forest Meteor. 2:16-23.
Yun, J. I., Cho, K. S., Hwang, H., Park, E. W. and Cho, S. I. 1998. Estimating microclimatic elements of a fully developed paddy rice canopy based on standard weather data. Korean J. Meteor. 34:216-221.

Yun, J. I., Yi, D. S., Choi, J. Y., Cho, S. I., Park, E. W. and Hwang, H. 1999. Elevation-corrected spatial interpolation for near-real time generation of meteorological surfaces from point observations. AgroInformatics J. 1:28-33. 